\title{
Phase II Trial of Neoadjuvant Gemcitabine and Cisplatin in Patients with Resectable Bladder Carcinoma
}

\author{
Daniel Herchenhorn, Rodrigo Dienstmann, Fabio A. Peixoto, Franz S. de Campos, Valdelice O. \\ Santos, Denise M. Moreira, Hedilene Cardoso, Isabele A. Small, Carlos G. Ferreira
}

Departments of Clinical Oncology (DH, FAP), Clinical Cancer Research (RD, VOS, IAS, CGF), Urology (FSC) and Radiology (DMM), National Cancer Institute, and Eli Lilly Medical Support (HC), Rio de Janeiro, RJ, Brazil

\begin{abstract}
Objectives: Gemcitabine and cisplatin (GC) is an active combination in the treatment of metastatic bladder cancer. We have prospectively analyzed the efficacy and tolerability of GC as neoadjuvant treatment of invasive bladder cancer.

Materials and Methods: In this single-institution phase II trial, patients with muscle-invasive transitional cell carcinoma received three cycles of gemcitabine $1200 \mathrm{mg} / \mathrm{m}^{2}$ on days 1 and 8 with cisplatin $75 \mathrm{mg} / \mathrm{m}^{2}$ on day 1 prior to surgery. Radiologic response was evaluated by computed tomography and magnetic resonance imaging. All patients were referred to surgery after chemotherapy completion.

Results: Between June 2002 and March 2005, 22 patients (19 males) were enrolled. Median age was 63 years. Initial stage was II (T2) in 11 and III (T3-4) in 11 patients. Median follow-up is 26 months (4-43). Partial or complete radiologic response rate was documented in 13 out of 20 assessable patients (70\%). One patient was excluded due to sarcomatoid carcinoma at definitive pathologic examination. Cystectomy was performed in 15 patients and pelvic radiotherapy in four patients. Nine out of 21 patients (43\%) relapsed and four (19\%) died due to disease progression. Complete pathologic response was observed in four patients (26.7\% of 15). Median progression-free survival was 27 months (CI 95\% not reached) with median overall survival of 36 months (CI 95\%: 28.7 - 43.3). Grade III/IV toxicity was infrequent, with no deaths due to chemotherapy. Conclusions: The combination of GC is effective and well-tolerated when used as neoadjuvant therapy in muscle-invasive bladder cancer. Longer follow-up is necessary to evaluate its impact on the overall survival of these patients.
\end{abstract}

Key words: bladder neoplasms; neoadjuvant therapy; cisplatin; gemcitabine Int Braz J Urol. 2007; 33: 630-8

\section{INTRODUCTION}

Bladder carcinoma is the second most prevalent genitourinary tract neoplasm in Brazil, with estimated 2000 deaths each year, which represents $1.7 \%$ of all cancer deaths (1). Radical cystectomy represents the standard of care in muscle invasive bladder cancer. Nevertheless, only about 50 to $60 \%$ of these patients will be cured with surgery alone (2). Therefore, there is a need for additional therapeutic modalities as an adjunct to local treatment in order to improve the outcome of patients with invasive bladder carcinoma. 
Systemic chemotherapy is active in advanced bladder cancer, with objective response rates in more than $50 \%$ of patients treated with MVAC (combination of methotrexate, vinblastine, adriamycin and cisplatin) (3). It has been administered also in the neoadjuvant and adjuvant settings in patients with bladder cancer at high risk of relapse $(4,5)$. The administration of preoperative chemotherapy has the advantage of immediate treatment of microscopic disease and better tolerability when compared with postsurgical treatment. Another major benefit of the neoadjuvant approach is the ability to assess the response of the primary lesion, which is of prognostic significance. This was illustrated in a report of 125 patients enrolled in multiple trials of cisplatin-based neoadjuvant therapy followed by definitive surgery (6). At a median follow-up of 25 months, $91 \%$ of complete responders (defined as $\leq$ pT1 at cystectomy) were disease-free, in contrast to only $37 \%$ of nonresponders ( $\geq$ pT2 at cystectomy). In addition, a survival benefit for neoadjuvant cisplatin-based chemotherapy was demonstrated in a 2005 Cochrane database review that included individual patient data from more than 3,000 individuals enrolled in 11 randomized trials comparing neoadjuvant chemotherapy with local therapy alone (7). Neoadjuvant cisplatin-based combination chemotherapy resulted in a significant $14 \%$ reduction in the risk of death, which translated into a 5\% absolute improvement in five-year overall survival (from $45 \%$ to $50 \%$ ).

Although regimens like MVAC are very active, toxicity has been a major concern and limits its clinical use: grade 3 or 4 mucositis is reported in more than $20 \%$ of patients, neutropenic sepsis in more than $10 \%$ and with toxic death rate of 3 to $4 \%(8,9)$. Combinations of cisplatin and novel agents have shown promising results in advanced disease. Gemcitabine plus cisplatin (GC) was associated with a similar response rate ( $49 \%$ versus $46 \%$ ), progression-free survival (7.7 versus 8.3 months), median survival (14 versus 15.2 months) and markedly less toxicity, when compared to MVAC chemotherapy, respectively $(10,11)$. The approval of this combination in the metastatic setting justifies its study as neoadjuvant treatment. The objective of this phase II trial is to evaluate the clinical and pathological response rates of patients with resectable bladder cancer treated with neoadjuvant GC and to assess the toxicity of this regimen in this setting.

\section{MATERIALS AND METHODS}

Patients - This is a phase II, nonrandomized, single-institution trial. Patients aged 18 to 70 years, Performance Status (Eastern Cooperative Oncology Group - ECOG) 0 - 2, with histologically confirmed muscle-invasive, resectable (clinical stages T2 - T4a, N0 - 1, M0) transitional cell carcinoma of the bladder and measurable disease on computed tomography (CT) or magnetic resonance imaging (MRI), were included in this trial. Patients should have adequate bone marrow function (hemoglobin $>10 \mathrm{~g} / \mathrm{dL}$, white blood cell count $>3.0 \times 10^{9} / \mathrm{L}$, absolute granulocyte count $>1.5 \times 10^{9} / \mathrm{L}$, platelet count $>150 \times 10^{9} / \mathrm{L}$ ), renal function (serum creatinine $<1.3 \mathrm{mg} / \mathrm{dL}$ and/or estimated creatinine clearance with Crockoft Formula $>60 \mathrm{~mL} / \mathrm{min}$ ), hepatic function (bilirubin $<1.2 \mathrm{mg} / \mathrm{dL}$, transaminases and alkaline phosphatase $<1.5 \mathrm{x}$ upper normal limit) and absence of concurrent disease precluding surgery. No previous chemotherapy or radiotherapy was allowed. Patients with preexisting peripheral neuropathy, previous history of cancer (except non melanoma skin cancer or in situ cervical cancer) were not eligible for this study.

Treatment schedule - Initial clinical staging included chest X-ray (and CT of the thorax in case of $\mathrm{X}$-ray abnormalities), abdominal and pelvic $\mathrm{CT}$, pelvic MRI, cystoscopy and biopsies of visible tumor. After 3 cycles of chemotherapy the patients were restaged. If operable, radical cystectomy and pelvic lymphadenectomy with or without neobladder reconstruction was offered within 6 weeks after the last course. For those who denied surgery or were not eligible for radical cystectomy and pelvic lymphadenectomy, radiation therapy was offered. Follow-up in the first 3 years included regular clinical assessment (every 2 months) and radiological examination (thorax X-ray and abdominal/pelvic CT every 6 months).

Patients were treated in an outpatient basis. Toxicity was graded according to National Cancer Institute Common Toxicity Criteria version 2.0. Che- 
motherapy was given for 3 cycles. Gemcitabine 1200 $\mathrm{mg} / \mathrm{m}^{2}$ was given by intravenous infusion in $250 \mathrm{~mL}$ saline solution over 30 minutes on days 1 and 8 of a 21-day cycle. Cisplatin $75 \mathrm{mg} / \mathrm{m}^{2}$ was given by intravenous infusion in $500 \mathrm{~mL}$ of normal saline over 60 minutes on day 1 of the cycle. Intravenous hydration and mannitol were given with each cisplatin infusion. All patients received premedication with intravenous dexamethasone plus ondansetron and postmedication with oral dexamethasone and ondansetron for 3 days as emesis prophylaxis.

Dose adjustment at the start of a treatment cycle was as follows: (1) treatment was delayed by 1 week in patients with an absolute granulocyte count < $1.5 \times 10^{9} / \mathrm{L}$ or platelet count $<100 \times 10^{9} / \mathrm{L}$ (if no recovery occurred after 2 weeks, then the patient was removed from the study); (2) dose of gemcitabine on day 8 was reduced by $25 \%$ if absolute granulocyte count between 1.0 to $1.2 \times 10^{9} / \mathrm{L}$ or platelet count between 50 to $75 \times 10^{9} / \mathrm{L}$ and by $50 \%$ if absolute granulocyte count between 0.7 to $1.0 \times 10^{9} / \mathrm{L}$; (3) dose of gemcitabine on day 8 was omitted if absolute granulocyte count $<0.7 \times 10^{9} / \mathrm{L}$ or platelet count $<50 \times 10^{9}$ / $\mathrm{L}$; (4) treatment was delayed by 1 week if serum creatinine $>1.3 \mathrm{mg} / \mathrm{dL}$ (if no recovery occurred after 2 weeks, then the patient was removed from the study); (5) for patients with grade 3 or more nonhematologic toxicity, if no recovery was obtained with 3 weeks delay, the patient was removed from the study (except for nausea, vomiting or alopecia); (6) for patients with recovered grade 3 or more nonhematologic toxicity, both gemcitabine and cisplatin doses were reduced by $25 \%$. Prophylactic use of growth factors was not permitted. Supportive care could include blood transfusions.

Treatment evaluation - Primary endpoint of this trial was to evaluate radiological and pathological response rate with the GC used in the neoadjuvant setting. Secondary endpoints included tolerability of the combination and evaluation of progression-free survival (PFS) and overall survival (OS). Radiological response criteria were as follows: (1) complete response was defined as the disappearance of all known disease at radiological examination (2) partial response was defined as a decrease in more than $30 \%$ in the longest diameter of the primary lesion on CT and MRI; (3) progressive disease was defined as increase in more than $20 \%$ in longest diameter of the primary lesion; (4) stable disease was defined as no established criteria for disease progression or partial response. Pathological complete response was defined as no residual disease at pathological examination, including patients with residual carcinoma in situ.

Progression-free survival was calculated from the day of treatment initiation until death or progression. Patients who were alive and who had not experienced disease progression were censored for progression-free survival at the date that they were last known to be alive or progression-free. Survival was measured from the date of treatment initiation until death. Patients who had not died were censored for overall survival when they were last known to be alive.

Statistical considerations - The planned sample size of the trial was 35 patients. All analyses were performed using SSPS statistical software. Survival curves for PFS and OS data were based on the Kaplan-Meier method. The study protocol was approved by the Ethics Committee of our institution and each patient signed a written informed consent.

\section{RESULTS}

Patients - Between June 2002 and March 2005, 22 patients were entered into the study. Accrual was poor due to overestimation of potentially eligible patients in our institution, as most of them either were older than 70 years at the time of diagnosis or had superficial disease. One of these 22 patients was not considered for response and toxicity evaluation because of protocol violation (sarcomatoid carcinoma at pathological review). Baseline characteristics are shown in Table-1. Median age at diagnosis was 63 years. All patients had Performance Status ECOG 0 or 1. Extravesical disease extension (T3 or T4) was found in 10 patients (48\%). Grade 3 tumors were found in all cases. All but 3 patients were current or former smokers $(86 \%)$.

Chemotherapy - Twenty patients received 3 cycles of neoadjuvant gemcitabine and cisplatin and 1 patient received only one cycle (protocol treatment 
Table 1 - Baseline characteristics.

\begin{tabular}{lll}
\hline & Number & $\%$ \\
\hline Age & & \\
$\quad$ Median & 64 & \\
$\quad$ Range & $48-68$ & \\
Gender & & \\
Male & 18 & $85.7 \%$ \\
Female & 3 & $14.3 \%$ \\
Initial clinical stage & & \\
T2 & 11 & $52.4 \%$ \\
T3 & 2 & $9.5 \%$ \\
T4 & 8 & $38.1 \%$ \\
\hline
\end{tabular}

was interrupted due to renal toxicity - persistent creatinine elevation higher than upper normal limit). Only in 4 cycles (from a total of $61-6.5 \%$ ) it was necessary to delay chemotherapy by one week due to neutropenia $(n=2)$, renal toxicity $(n=1)$ or infection $(n=$ $1)$. Nineteen patients received all full doses of chemotherapy and only 1 patient required $25 \%$ reduction in the dose of gemcitabine on day 8 of 2 cycles due to neutropenia. Toxicity was mild and only $38 \%$ of the patients presented grade 3 or 4 hematologic toxicity, as shown in Table-2. One patient required red blood cell transfusion and there was 1 episode of uncomplicated febrile neutropenia. Other grade 3 toxicities included nausea (28.6\%) and rash (1 patient).

Postchemotherapy evaluation and treatment - As shown in Table-3, radical cystectomy was the definitive treatment of 15 patients. Median time between last chemotherapy cycle and surgery was 47 days (31 - 177). Two patients had delayed surgery not related to neoadjuvant treatment toxicity. Three patients refused surgery and 1 had a myocardial infarction in the preoperative week. No evidence of inoperability was detected in these 4 patients that had radiation therapy to the pelvis as definitive treatment ( $45 \mathrm{~Gy}$ in the pelvis with $20 \mathrm{~Gy}$ boost to bladder). One patient refused any definitive treatment and another progressed systemically before surgery.

Cystectomy was complicated by wound infection and pyelonepritis in 1 patient, urinary fistula requiring laparotomy in 1 patient and hypertensive crisis followed by cardiac failure and fatal arrhythmia in 1 patient with prior history of cardiac disease.

Radiological response was assessable in 20 patients (1 patient had unmeasurable disease at initial radiological examination). Evaluation with $\mathrm{CT}$ was complemented by MRI and is presented in Table- 4 . Most patients had partial response to treatment (65\%).

Table 2 - Toxicity of gemcitabine plus cisplatin: worst toxicity by patient $(n=21)$.

\begin{tabular}{|c|c|c|c|c|c|}
\hline & Grade 0 & Grade 1 & Grade 2 & Grade 3 & Grade 4 \\
\hline \multicolumn{6}{|l|}{ Hematologic } \\
\hline Granulocytes & 8 & 3 & 3 & 6 & 1 \\
\hline Platelets & 18 & 2 & 0 & 1 & 0 \\
\hline Hemoglobin & 0 & 18 & 3 & 0 & 0 \\
\hline Alopecia & 9 & 11 & 1 & - & - \\
\hline Fatigue & 8 & 10 & 3 & 0 & 0 \\
\hline Infection & 13 & 2 & 5 & 1 & 0 \\
\hline Nausea & 1 & 6 & 8 & 6 & - \\
\hline Vomiting & 7 & 8 & 6 & 0 & 0 \\
\hline Neuropathy (sensory) & 16 & 2 & 3 & 0 & 0 \\
\hline Fever & 19 & 2 & 0 & 0 & 0 \\
\hline \multicolumn{6}{|l|}{ Dermatologic } \\
\hline Rash & 18 & 2 & 0 & 1 & 0 \\
\hline Injection site reaction & 20 & 1 & 0 & 0 & - \\
\hline Weight loss & 18 & 3 & 1 & 0 & - \\
\hline
\end{tabular}


Table 3 - Post-chemotherapy treatment $(n=21)$.

\begin{tabular}{lrc}
\hline & N & $\%$ \\
\hline $\begin{array}{l}\text { Surgery } \\
\quad \text { Bricker } \\
\quad \text { Ileal neobladder }\end{array}$ & 15 & $71.4 \%$ \\
$\begin{array}{l}\text { Radiation therapy } \\
\text { Refused definitive treatment }\end{array}$ & 6 & \\
$\begin{array}{l}\text { Progressed systemically before } \\
\text { definitive treatment }\end{array}$ & 1 & $4.75 \%$ \\
\hline
\end{tabular}

Table 4 - Radiological response rate $(n=20)$.

\begin{tabular}{lcc}
\hline & N & \% \\
\hline Complete response & 1 & $5.0 \%$ \\
Partial response & 13 & $65.0 \%$ \\
Stable disease & 2 & $10.0 \%$ \\
Progressive disease & 4 & $20.0 \%$ \\
\hline
\end{tabular}

Post-chemotherapy pathological stage for those submitted to surgery is shown in Table-5. No microscopic evidence of tumor was found in 3 patients and carcinoma in situ in 1 patient (pathological complete response in 4 out of 15 patients - 26.7\%). Median number of lymph node dissected was $11(0-25)$. Three patients had metastasis to pelvic lymph nodes and one patient had positive surgical margins.

Progression-free survival and overall survival - Median follow-up was 26 months (4 - 43). During the follow-up, 9 patients relapsed (42.8\%). Median estimated PFS was 27 months (95\% CI: not reached), as shown in Figure-1. Six patients had systemic relapse, 2 concomitant locoregional and systemic relapse and 1 exclusive locoregional progression. From these
Table 5 - Post-chemotherapy (surgical) pathological stage.

\begin{tabular}{ccc}
\hline & $\mathbf{N}$ & $\mathbf{\%}$ \\
\hline Stage 0 & 3 & 14.3 \\
Stage 0is & & \\
Tis N0 & 1 & 4.75 \\
Stage I & & \\
T1N0 & 1 & 4.75 \\
Stage II & 3 & 14.3 \\
T2aN0 & 2 & 9.50 \\
T2bN0 & 1 & 4.75 \\
Stage III & 4 & 18.0 \\
T3aN0 & 2 & 9.50 \\
T3bN0 & 1 & 4.75 \\
T4aN0 & 1 & 4.75 \\
Stage IV & 3 & 14.3 \\
T1 N2 & 1 & 4.75 \\
T3bN2 & 1 & 4.75 \\
T4aN2 & 1 & 4.75 \\
Not available & 6 & 28.6 \\
\hline
\end{tabular}

patients, 4 received second-line chemotherapy, 4 are dead and 5 are still on palliative treatment.

During follow-up, 6 patients died: 4 due to bladder cancer relapse, 1 due to postoperative complications and another due to myocardial infarction with no clinical or radiological evidence of disease progression. Median estimated overall survival was 36 months (95\% CI: 28.7 - 43.3), as shown in Figure-2.

\section{COMMENTS}

The administration of neoadjuvant chemotherapy in patients with operable bladder cancer has been previously studied in randomized trials. The largest trial was performed jointly by the Medical Research Council and the European Organization for Research and Treatment of Cancer (EORTC) (12). Patients with high-grade muscle-invasive bladder transitional cell carcinoma were randomly assigned to three cycles of neoadjuvant CMV (cisplatin, methotrexate, and vinblastine, $n=491)$ or no chemotherapy ( $n=$ 


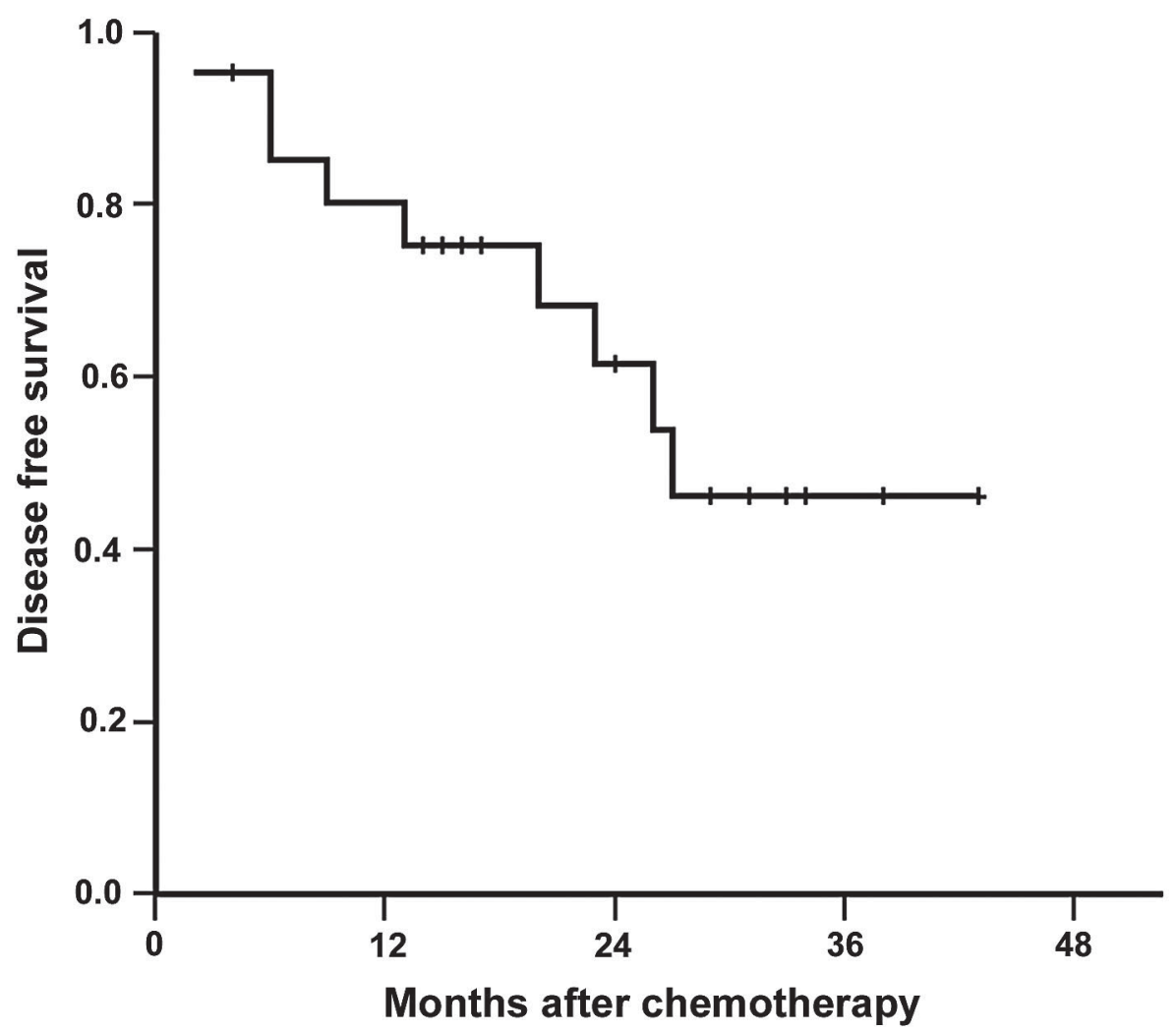

Figure 1 - Progression-free survival of 21 patients treated with neoadjuvant gemcitabine and cisplatin for invasive bladder cancer.

485), followed by each institution's choice of local management (radical cystectomy or radiation therapy). The pathological complete response rate in the neoadjuvant group was $33 \%$, and the absolute OS benefit from chemotherapy at three years was $5.5 \%$ (55.5\% versus $50 \%$ ), which was not significant. A later report with seven years of follow-up demonstrated a $15 \%$ OS benefit with neoadjuvant CMV, but it was still not statistically significant (13).

A sufficiently powered United States Intergroup trial randomly assigned 317 patients with T2-4a N0 bladder cancer to cystectomy with or without three preoperative courses of MVAC (4). In the final report, with a median follow-up of 8.7 years, patients treated with MVAC were significantly more likely to have a pathological complete response (38\% versus $15 \%)$. The improvements in median OS (77 versus 46 months, $\mathrm{p}=0.06$ ), and five year OS (57\% versus $43 \%, p=0.06)$ were of borderline statistical signifi- cance. These results were obtained after a median follow-up of 8.7 years.

Our trial is the first complete report on the feasibility and efficacy of the combination of gemcitabine and cisplatin as neoadjuvant treatment of invasive bladder carcinoma. The results confirm the excellent tolerability of this regimen and show that it can be safely administered in this setting. Three cycles of this combination resulted in mild toxicity and was completed by $95 \%$ of patients. This seems promising compared to neoadjuvant MVAC, which is associated with severe gastrointestinal toxicity in one-third of the patients and grade $3 / 4$ hematological adverse effects in at least $60 \%$ of the patients (4). Chemotherapy resulted in high percentage of complete/ partial radiological response (70\%) and in 4 out of 15 patients submitted to radical cystectomy there was complete pathological response (26.7\%). Grossman et al. found $38 \%$ of complete pathological response with MVAC chemotherapy (4). Re- 


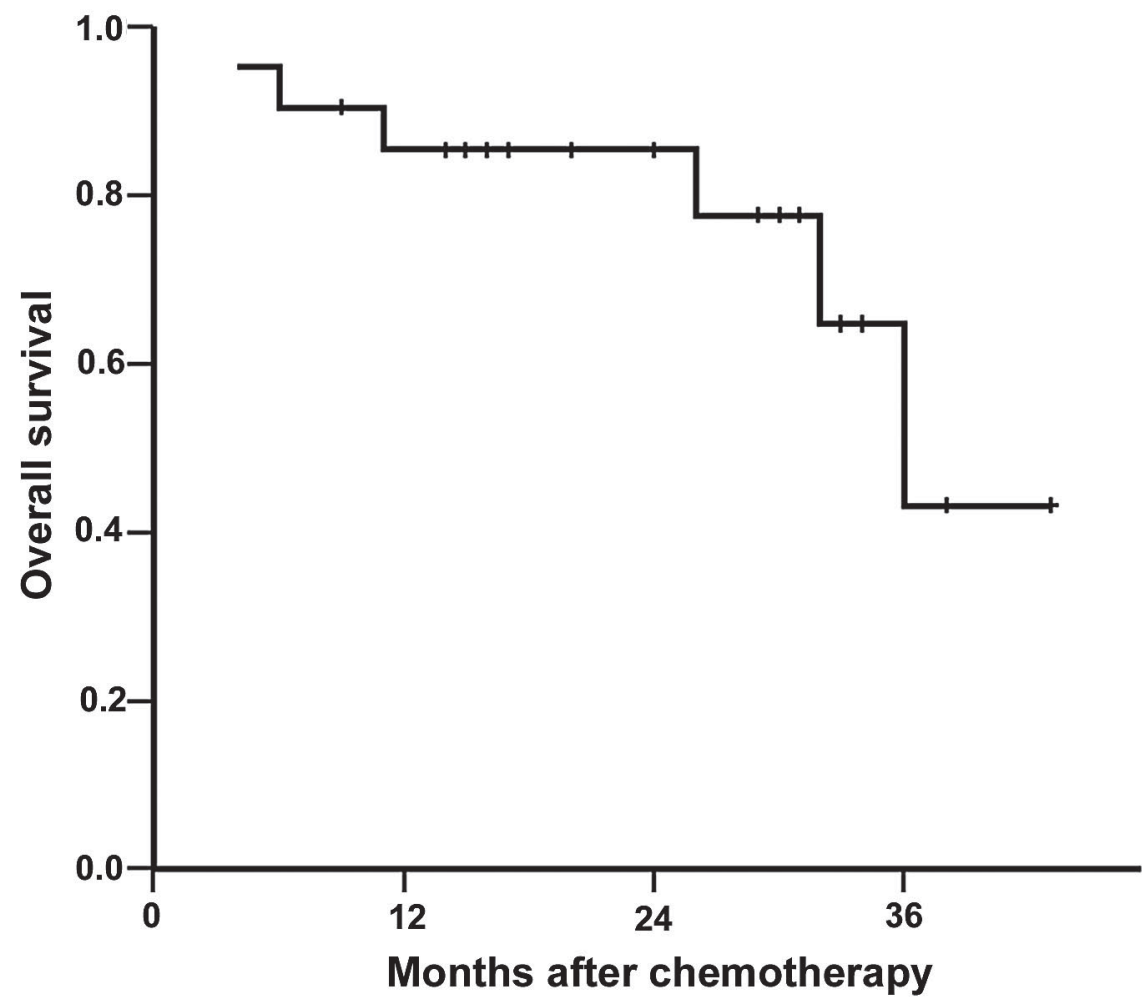

Figure 2 - Overall survival of 21 patients treated with neoadjuvant gemcitabine and cisplatin for invasive bladder cancer.

garding survival data, our trial has a limited follow-up of 2.2 years, what justifies the unfavorable median estimated survival of 36 months, as compared to the Intergroup trial (4).

In our trial, nearly $70 \%$ of the randomized patients were submitted to radical cystectomy, what is consistent with previous studies evaluating neoadjuvant chemotherapy for invasive bladder cancer (4). Median time from last course of chemotherapy and surgery was 6 weeks, what is in agreement with the protocol schedule. However, extension of lymph node dissection was not uniform and half of the patients had less than 10 nodes removed, what is known to be associated with shorter postcystectomy survival (14). Herr et al. showed that surgical variables associated with longer survival included negative margins and more than 10 lymph nodes removed, using a multivariate model adjusted for neoadjuvant chemotherapy, age, pathologic stage and node status. These results emphasize the importance of surgery in the treatment of bladder cancer.

Other studies have reported initial results with neoadjuvant GC in invasive bladder cancer. Bolotina et al. presented in abstract form the results of another phase II trial of neoadjuvant GC in patients with transitional bladder carcinoma (15). Gemcitabine $1000 \mathrm{mg} /$ $\mathrm{m}^{2}$ was given on days 1,8 and 15 and cisplatin 100 $\mathrm{mg} / \mathrm{m}^{2}$ on day 2 , for 2 cycles. Complete pathological response was achieved in 6 out of 20 patients (30\%) and partial response in 9 patients (45\%). No grade 3 or 4 toxicities were observed in the trial.

Additionally, Khaled et al. are conducting a phase III randomized trial of neoadjuvant GC compared to radical cystectomy alone in patients with bladder cancer in Egypt (16). In patients with complete response to neoadjuvant treatment, additional chemotherapy and radiation therapy was recommended in order to preserve the bladder. Radical cystectomy was 
recommended to patients with partial response. Most patients had squamous cell carcinoma (59 of 114). Treatment was well tolerated and bladder preservation was feasible in 11 out of 58 patients randomized to neoadjuvant chemotherapy. Although data of this trial cannot be compared with our results once bladder squamous cell carcinomas respond differently to chemotherapy, it shows that effective neoadjuvant treatment might be used for selection of patients for bladder preservation. Sample size and follow-up are also still insufficient for survival evaluation.

Our results suggest that GC might be an attractive alternative to MVAC, as previously reported in the metastatic setting. Nevertheless, it should be noted the small sample size and median follow-up of our trial. Therefore, for definitive conclusions we should wait properly designed randomized trials.

\section{CONFLICT OF INTEREST}

Eli Lilly Company funded the trial.

\section{REFERENCES}

1. Instituto Nacional de Câncer do Brasil: Estimativa de Incidência e Mortalidade por Câncer no Brasil. Rio de Janeiro (Brazil): INCA; 2006

2. Shipley WU, Kaufman DS, McDougal WS, et al.: Cancers of the genitourinary system. In DeVita VT, Hellman S, Rosenberg SA (eds.), Cancer: Practice and Principles in Oncology. 7th ed., Philadelphia, Lippincot and Williams. 2004.

3. Moore MJ, Winquist EW, Murray N, Tannock IF, Huan $\mathrm{S}$, Bennett K, et al.: Gemcitabine plus cisplatin, an active regimen in advanced urothelial cancer: a phase II trial of the National Cancer Institute of Canada Clinical Trials Group. J Clin Oncol. 1999; 17: 2876-81.

4. Grossman HB, Natale RB, Tangen CM, Speights VO, Vogelzang NJ, Trump DL, et al.: Neoadjuvant chemotherapy plus cystectomy compared with cystectomy alone for locally advanced bladder cancer. $\mathrm{N}$ Engl J Med. 2003; 349: 859-66.

5. Stockle M, Meyenburg W, Wellek S, Voges G, Gertenbach U, Thuroff JW, et al.: Advanced bladder cancer (stages pT3b, pT4a, pN1 and pN2): improved survival after radical cystectomy and 3 adjuvant cycles of chemotherapy. Results of a controlled prospective study. J Urol. 1992; 148: 302-7.

6. Splinter TA, Scher HI, Denis L, Bukowski R, Simon S, Klimberg I, et al.: The prognostic value of the pathological response to combination chemotherapy before cystectomy in patients with invasive bladder cancer. European Organization for Research on Treatment of Cancer-Genitourinary Group. J Urol. 1992; 147: 606-8.

7. Advanced Bladder Cancer Overview Collaboration: Neoadjuvant chemotherapy for invasive bladder cancer. Cochrane Database Syst Rev 2005; 2: CD005246.

8. Loehrer PJ Sr, Einhorn LH, Elson PJ, Crawford ED, Kuebler P, Tannock I, et al.: A randomized comparison of cisplatin alone or in combination with methotrexate, vinblastine, and doxorubicin in patients with metastatic urothelial carcinoma: a cooperative group study. J Clin Oncol. 1992; 10: 1066-73.

9. Sternberg CN, Yagoda A, Scher HI, Watson RC, Geller $\mathrm{N}$, Herr HW, et al.: Methotrexate, vinblastine, doxorubicin, and cisplatin for advanced transitional cell carcinoma of the urothelium. Efficacy and patterns of response and relapse. Cancer. 1989; 64: 2448-58.

10. von der Maase H, Hansen SW, Roberts JT, Dogliotti L, Oliver T, Moore MJ, et al.: Gemcitabine and cisplatin versus methotrexate, vinblastine, doxorubicin, and cisplatin in advanced or metastatic bladder cancer: results of a large, randomized, multinational, multicenter, phase III study. J Clin Oncol. 2000; 18: 3068-77.

11. von der Maase H, Sengelov L, Roberts JT, Ricci S, Dogliotti L, Oliver T, et al.: Long-term survival results of a randomized trial comparing gemcitabine plus cisplatin, with methotrexate, vinblastine, doxorubicin, plus cisplatin in patients with bladder cancer. J Clin Oncol. 2005; 23: 4602-8.

12. No authors listed: Neoadjuvant cisplatin, methotrexate, and vinblastine chemotherapy for muscle-invasive bladder cancer: a randomised controlled trial. International collaboration of trialists. Lancet. 1999; 354: 533-40.

13. Hall RR: Updated results of a randomised controlled trial of neoadjuvant cisplatin (C), methotrexate $(\mathrm{M})$ and vinblastine (V) chemotherapy for muscle-invasive bladder cancer. Proc Am Soc Clin Oncol. 2002; 21: Abstract 710.

14. Herr HW, Faulkner JR, Grossman HB, Natale RB, deVere White R, Sarosdy MF, et al.: Surgical factors influence bladder cancer outcomes: a cooperative group report. J Clin Oncol. 2004; 22: 2781-9.

15. Bolotina L, Koroleva A, Trophimova A: Short-term results of neoadjuvant chemotherapy with gemcitabine 
and cisplatin in invasive bladder cancer. Proc Am Soc Clin Oncol. 2003; 22: Abstract 1679.

16. Khaled HM, Zaghloul MS, Ghoneim RA, Saber RA, Manie M, Enin HM, at al.: Gemcitabine and cisplatin as

Correspondence address:

Dr. Carlos G. Ferreira

Rua André Cavalcanti, 37

Rio de Janeiro, RJ, 20231-050, Brazil

Fax: + 55 213233-1411

E-mail: cferreira@inca.gov.br neoadjuvant chemotherapy for invasive bladder cancer: Effect on bladder preservation and survival. Proc Am Soc Clin Oncol. 2003; 22: Abstract 1652.

March 30, 2007

\section{EDITORIAL COMMENT}

Neoadjuvant chemotherapy is an exciting new area of research in the treatment of bladder cancer. It has been previously described that gemcitabine and cisplatin (GC) offers less toxicity than MVAC in both the neoadjuvant and adjuvant setting.

Clearly, there is inadequate statistical power in this study to determine meaningful clinical endpoints. However, the authors do recognize this and address it appropriately in the conclusions section. GC is again well-tolerated in this study; however, this is a known fact.

The authors provided an excellent explanation of lymph nodes obtained and inclusion of the importance of an extended dissection. This is a key point whether one is discussing chemotherapy in the neoadjuvant or adjuvant setting.
I would caution the authors inclusion of the study by Khaled et al (Reference 16) given the high number of patients with primary squamous cell carcinoma (over 50\%!). Transitional cell carcinoma responds differently to chemotherapy in general. The statement that this trial shows that neoadjuvant chemotherapy is effective in this population is true. However, it should not be compared with patients who have primary TCC of the bladder.

I am unsure how to word the fact that the scientific community will certainly not base management decisions on such an underpowered study. However, it does promote a basis for continued research and improvements in neoadjuvant chemotherapy in bladder cancer. At the least, it reinforces the need for a large trial evaluating the proposed clinical endpoints.

Dr. Eric S. Gwynn Department of Urology

Wake Forest University School of Medicine Winston-Salem, North Carolina, USA E-mail:egwynn@wfubmc.edu 